Pacific Northwest

National Laboratory

Operated by Battelle for the

U.S. Department of Energy

\section{Hanford Site Annual Treatability Studies Report - Calendar Year 2000}

\author{
M. W. McCoy
}

February 2001

Prepared for the U.S. Department of Energy under Contract DE-AC06-76RL01830 


\title{
DISCLAIMER
}

This report was prepared as an account of work sponsored by an agency of the United States Government. Neither the United States Government nor any agency thereof, nor Battelle Memorial Institute, nor any of their employees, makes any warranty, express or implied, or assumes any legal liability or responsibility for the accuracy, completeness, or usefulness of any information, apparatus, product, or process disclosed, or represents that its use would not infringe privately owned rights. Reference herein to any specific commercial product, process, or service by trade name, trademark, manufacturer, or otherwise does not necessarily constitute or imply its endorsement, recommendation, or favoring by the United States Government or any agency thereof, or Battelle Memorial Institute. The views and opinions of authors expressed herein do not necessarily state or reflect those of the United States Government or any agency thereof.

\author{
PACIFIC NORTHWEST NATIONAL LABORATORY \\ operated by \\ BATTELLE \\ for the \\ UNITED STATES DEPARTMENT OF ENERGY \\ under Contract DE-ACO6-76RL0183O
}

Printed in the United States of America
Available to DOE and DOE contractors from the Office of Scientific and Technical Information,
P.O. Box 62, Oak Ridge, TN 37831-0062;
ph: (865) 576-8401
fax: (865) 576-5728
email: reports@adonis.osti.gov

\footnotetext{
Available to the public from the National Technical Information Service,

U.S. Department of Commerce, 5285 Port Royal Rd., Springfield, VA 22161 ph: (800) 553-6847 fax: (703) 605-6900 email: orders@ntis.fedworld.gov

online ordering: http://www.ntis.gov/support
}

This document was printed on recycled paper. 


\section{Hanford Site Annual Treatability Studies Report - Calendar Year 2000}

Prepared for

the U.S. Department of Energy

under Contract DE-AC06-76RL01830

Pacific Northwest National Laboratory

Richland, Washington 99352

United States

Department of Energy

Richland, Washington

Approved for Public Release 


\section{Executive Summary}

This report provides information required to be reported annually by the Washington Administrative Code (WAC) 173-303-071 (3)(r)(ii)(F) and (3)(s)(ix) on the treatability studies conducted on the Hanford Site in 2000. These studies were conducted as required by WAC 173-303-071, "Excluded Categories of Waste," sections (3)(r) and (s).

Unless otherwise noted, the waste samples were provided by and the treatability studies were performed for the U.S. Department of Energy, Richland Operations Office, P.O. Box 550, Richland, Washington 99352. The U.S. Environmental Protection Agency identification number for these studies is WA7890008967. 


\section{Contents}

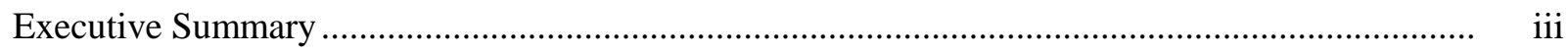

Table 1. 2000 Annual Report For Small-Quantity Treatability Studies Conducted By

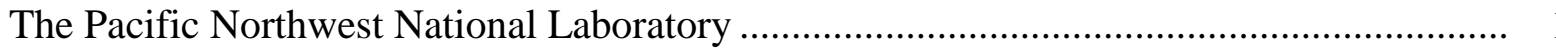

Table 2. 2000 Annual Report For Small-Quantity Treatability Studies Conducted By

Fluor Hanford and Its Major Subcontractors ................................................................. 
Table 1. 2000 Annual Report For Small-Quantity Treatability Studies Conducted By The Pacific Northwest National Laboratory

\begin{tabular}{|c|c|c|c|c|c|c|c|c|c|c|}
\hline Location & $\begin{array}{l}\text { Waste } \\
\text { Type }\end{array}$ & $\begin{array}{c}\text { Total Quantity in } \\
\text { Storage (Daily) }\end{array}$ & $\begin{array}{c}\text { Technology } \\
\text { Tested }\end{array}$ & $\begin{array}{c}\text { Dates of } \\
\text { Study }\end{array}$ & $\begin{array}{c}\text { Amount } \\
\text { Tested } \\
\text { Jan.-Dec. } \\
2000 \\
(\mathbf{K g}) \\
\end{array}$ & $\begin{array}{c}\text { Amount to } \\
\text { Be Tested } \\
\text { Jan.-Dec. } \\
2001 \\
(\mathrm{Kg}) \\
\end{array}$ & $\begin{array}{c}\text { Final } \\
\text { Disposal } \\
\text { of Sample } \\
\text { Portion } \\
\end{array}$ & $\begin{array}{c}\text { Final } \\
\text { Disposal } \\
\text { of } \\
\text { Residues }\end{array}$ & $\begin{array}{c}\begin{array}{c}\text { Amount } \\
\text { of } \\
\text { Sample } \\
\text { Shipped } \\
\text { (Kg) }\end{array} \\
\end{array}$ & $\begin{array}{c}\text { Date of } \\
\text { Shipment }\end{array}$ \\
\hline 325 & $\begin{array}{c}\text { Tank Waste } \\
\text { (AN-102, } \\
\text { AY-102, } \\
\text { AP-101, C- } \\
104)\end{array}$ & $\begin{array}{c}0 \mathrm{Kg} \\
1 / 1 / 00-2 / 9 / 00 \\
3 \mathrm{Kg} \\
2 / 10 / 00-2 / 14 / 00 \\
0 \mathrm{Kg} \\
2 / 15 / 00-11 / 8 / 00 \\
4.6 \mathrm{Kg} \\
11 / 9 / 00-11 / 12 / 00 \\
9.2 \mathrm{Kg} \\
11 / 13 / 00-11 / 14 / 00 \\
13.9 \mathrm{Kg} \\
11 / 15 / 00-11 / 16 / 00 \\
18.0 \mathrm{Kg} \\
11 / 17 / 00-11 / 26 / 00 \\
0 \mathrm{Kg} \\
11 / 27 / 00-12 / 31 / 00\end{array}$ & $\begin{array}{c}\text { Hanford } \\
\text { vitrification } \\
\text { process }\end{array}$ & $\begin{array}{l}2 / 15 / 00- \\
12 / 21 / 00\end{array}$ & 21.0 & 20 & NA & CWC & 0 & NA \\
\hline 325 & $\begin{array}{c}\text { Tank Waste } \\
\text { Supernate } \\
\text { (AW-101, } \\
\text { C-104, AZ- } \\
\text { 102) }\end{array}$ & $\begin{array}{c}0.4 \mathrm{Kg} \\
1 / 1 / 00-1 / 4 / 00 \\
0 \mathrm{Kg} \\
1 / 5 / 00-12 / 31 / 00\end{array}$ & $\begin{array}{l}\text { Cross-flow } \\
\text { filtration, ion } \\
\text { exchange, } \\
\text { and } \\
\text { vitrification }\end{array}$ & $\begin{array}{l}1 / 1 / 00- \\
9 / 29 / 00\end{array}$ & 0.4 & 0 & NA & CWC & 0 & NA \\
\hline
\end{tabular}


Table 1. (Contd.)

\begin{tabular}{|c|c|c|c|c|c|c|c|c|c|c|}
\hline Location & Waste Type & $\begin{array}{c}\text { Total Quantity in } \\
\text { Storage (Daily) }\end{array}$ & $\begin{array}{c}\text { Technology } \\
\text { Tested }\end{array}$ & $\begin{array}{c}\text { Dates of } \\
\text { Study }\end{array}$ & $\begin{array}{c}\text { Amount } \\
\text { Tested } \\
\text { Jan.-Dec. } \\
2000 \\
(\mathbf{K g})\end{array}$ & $\begin{array}{c}\text { Amount to } \\
\text { Be Tested } \\
\text { Jan.-Dec. } \\
2001 \\
(\mathrm{Kg})\end{array}$ & $\begin{array}{c}\text { Final } \\
\text { Disposal } \\
\text { of Sample } \\
\text { Portion }\end{array}$ & $\begin{array}{c}\text { Final } \\
\text { Disposal } \\
\text { of } \\
\text { Residues }\end{array}$ & 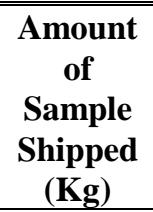 & $\begin{array}{c}\text { Date of } \\
\text { Shipment }\end{array}$ \\
\hline
\end{tabular}


Table 2. 2000 Annual Report For Small-Quantity Treatability Studies Conducted By Fluor Hanford and Its Major Subcontractors

\begin{tabular}{|c|c|c|c|c|c|c|c|c|c|c|}
\hline Location & $\begin{array}{c}\text { Waste } \\
\text { Type }\end{array}$ & $\begin{array}{c}\text { Total Quantity } \\
\text { in Storage } \\
\text { (Daily) }\end{array}$ & $\begin{array}{c}\text { Technology } \\
\text { Tested }\end{array}$ & $\begin{array}{c}\text { Dates of } \\
\text { Study }\end{array}$ & $\begin{array}{c}\text { Amount } \\
\text { Tested } \\
\text { Jan.-Dec. } \\
2000 \text { (or in } \\
\text { Process) } \\
\text { (Kg) }\end{array}$ & $\begin{array}{c}\text { Amount to } \\
\text { Be Tested } \\
\text { Jan.-Dec. } \\
\mathbf{2 0 0 1} \\
(\mathrm{Kg}) \\
\end{array}$ & $\begin{array}{c}\text { Final } \\
\text { Disposal of } \\
\text { Unused } \\
\text { Sample } \\
\text { Portion } \\
\end{array}$ & $\begin{array}{c}\text { Final } \\
\text { Disposal of } \\
\text { Residues } \\
\end{array}$ & $\begin{array}{c}\text { Amount of } \\
\text { Sample } \\
\text { Shipped } \\
(\mathbf{K g}) \\
\end{array}$ & $\begin{array}{c}\text { Date of } \\
\text { Shipment }\end{array}$ \\
\hline WSCF & $\begin{array}{l}\text { Radioactive } \\
\text { dilute } \\
\text { phosphoric } \\
\text { acid } \\
\text { solution }^{11}\end{array}$ & $\begin{array}{c}2.75 \mathrm{Kg} \\
1 / 1 / 00-1 / 13 / 00 \\
1 \mathrm{Kg} \\
1 / 14 / 00-4 / 30 / 00\end{array}$ & Grouting & $\begin{array}{l}1 / 1 / 00- \\
1 / 13 / 00\end{array}$ & $1.75 \mathrm{Kg}$ & 0 & NA & $\begin{array}{l}\text { Put into } \\
\text { archive. }\end{array}$ & 0 & NA \\
\hline & & $\begin{array}{c}2 \mathrm{Kg} \\
5 / 1 / 00-5 / 19 / 00 \\
0 \mathrm{Kg} \\
5 / 20 / 00\end{array}$ & Grouting & $\begin{array}{l}5 / 1 / 00- \\
5 / 19 / 00\end{array}$ & $2.00 \mathrm{Kg}$ & 0 & NA & $\begin{array}{l}\text { Put into } \\
\text { archive per } \\
\text { customer } \\
\text { request. }\end{array}$ & 0 & NA \\
\hline WSCF & $\begin{array}{l}\text { Treated } \\
\text { sample } \\
\text { residue of } \\
\text { radioactive } \\
\text { dilute } \\
\text { phosphoric } \\
\text { acid } \\
\text { solution }\end{array}$ & $\begin{array}{c}0 \mathrm{Kg} \\
1 / 1 / 00-1 / 13 / 00 \\
3.75 \mathrm{Kg}^{2} \\
1 / 14 / 005 / 19 / 00 \\
8.95 \mathrm{Kg}^{2} \\
5 / 20 / 00- \\
12 / 31 / 00\end{array}$ & N/A & N/A & NA & N/A & NA & $\begin{array}{l}\text { Put onto } \\
\text { archive per } \\
\text { customer } \\
\text { request. }\end{array}$ & 0 & NA \\
\hline
\end{tabular}

\footnotetext{
${ }^{1}$ Waste received from the Puget Sound Naval Shipyard, 1400 Farragut Ave, Bremerton, WA 98314, WA2170023418.

${ }^{2}$ Treated sample residue weight includes the acid solution and grout.
} 
Table 2. (Contd)

\begin{tabular}{|c|c|c|c|c|c|c|c|c|c|c|}
\hline Location & $\begin{array}{l}\text { Waste } \\
\text { Type }\end{array}$ & $\begin{array}{l}\text { Total Quantity in } \\
\text { Storage (Daily) }\end{array}$ & $\begin{array}{c}\text { Technology } \\
\text { Tested }\end{array}$ & $\begin{array}{l}\text { Dates of } \\
\text { Study }\end{array}$ & $\begin{array}{l}\text { Amount } \\
\text { Tested } \\
\text { Jan.-Dec. } \\
\text { 2000 (or in } \\
\text { Process) } \\
\text { (Kg) }\end{array}$ & $\begin{array}{c}\text { Amount to } \\
\text { Be Tested } \\
\text { Jan.-Dec. } \\
2001 \\
(\mathbf{K g})\end{array}$ & $\begin{array}{c}\text { Final } \\
\text { Disposal of } \\
\text { Unused } \\
\text { Sample } \\
\text { Portion }\end{array}$ & $\begin{array}{c}\text { Final } \\
\text { Disposal of } \\
\text { Residues }\end{array}$ & $\begin{array}{c}\text { Amount of } \\
\text { Sample } \\
\text { Shipped } \\
(\mathrm{Kg})\end{array}$ & $\begin{array}{c}\text { Date of } \\
\text { Shipment }\end{array}$ \\
\hline $272-W$ & $\begin{array}{c}\text { Mop water } \\
\text { with heavy } \\
\text { metals }\end{array}$ & $\begin{array}{c}0 \mathrm{Kg} \\
1 / 1 / 00-6 / 18 / 00 \\
200 \mathrm{Kg} \\
6 / 19 / 00-6 / 21 / 00 \\
400 \mathrm{Kg} \\
6 / 22 / 00-8 / 16 / 00 \\
600 \mathrm{Kg} \\
8 / 17 / 008 / 31 / 00 \\
800 \mathrm{Kg} \\
9 / 1 / 00-12 / 1 / 00 \\
799.5 \mathrm{Kg} \\
12 / 2 / 00-12 / 31 / 00\end{array}$ & $\begin{array}{l}\text { Filtration and } \\
\text { ion exchange } \\
\text { to remove } \\
\text { trace } \\
\text { elements of } \\
\text { metals }\end{array}$ & $\begin{array}{l}6 / 21 / 00 \\
8 / 22 / 00 \\
8 / 31 / 00\end{array}$ & $\begin{array}{c}200.0 \mathrm{Kg} \\
200.0 \mathrm{Kg} \\
4.0 \mathrm{Kg}\end{array}$ & $1000 \mathrm{Kg}$ & $\begin{array}{c}\text { In storage at } \\
272 \mathrm{~W} \text { for } \\
\text { eventual } \\
\text { disposal } \\
\text { pending } \\
\text { reuse in } \\
\text { Treatability } \\
\text { Study. }\end{array}$ & $\begin{array}{c}\text { In storage at } \\
272 \mathrm{~W} \\
\text { awaiting } \\
\text { offsite } \\
\text { disposal as } \\
\text { dangerous } \\
\text { waste. } \\
\text { Residue of } \\
\text { sample } \\
\text { shipped to } \\
\text { offsite lab } \\
\text { managed by } \\
\text { lab to be } \\
\text { manifested } \\
\text { to TSD. }\end{array}$ & $0.5^{(\mathrm{a})}$ & $12 / 1 / 00$ \\
\hline
\end{tabular}




\section{Distribution}

No. of

Copies

ONSITE

3 U.S. Department of Energy, Richland Operations
T.L. Aldridge
$\mathrm{K} 8-50$
T.L. Davis
K8-50
G.A. Williams
A5-15

4 Fluor Hanford

R.A. Del Mar

G3-26

W.E. Toebe

G1-37

J.J. Warwick

S3-25

J.A. Dawson

G1-27

10 Pacific Northwest National Laboratory

E.L. Grohs (5)

P8-34

J.D. Jacobsen

P8-34

H.T.Tilden

P8-34

Commitment Control

Technical Information and Report Files (2)

Distr. 1 